\title{
Wenn es im Frühling in unseren Wäldern bunt wird
}

Veit Martin Dörken \& Hilke Steinecke

\section{Abstract}

Unlike tropical evergreen forests, our native seasonal broad leaved forests are characterized by numerous spring geophytes, which are sprouting already in late winter and finish their annual life cycle already before the trees are enfolding their leaves. The conditions in our deciduous forests and a selection of native spring geophytes is presented here.

\section{Zusammenfassung}

In Gegensatz zu immergrünen tropischen Regenwäldern sind unsere heimischen sommergrünen Laubwälder durch eine Vielzahl an Frühlingsgeophyten geprägt, die bereits im Spätwinter austreiben und ihren jährlichen Lebenszyklus bereits zum Zeitpunkt des Laubaustriebs der Bäume abgeschlossen haben. Im Folgenden wird der Lebensbereich heimischer Laubwald und eine Auswahl an typischen Frühlingsgeophyten vorgestellt.

\section{Einleitung}

Unsere heimischen, winterkahlen Laubwälder sind durch einen jahreszeitlichen Wechsel in der Bodenvegetation gekennzeichnet. Bereits im Spätwinter treiben die ersten Frühlingsgeophyten wie das Scharbockskraut aus, denen dann noch zahlrei- che weitere Arten folgen und den Waldboden stellenweise in ein üppiges Blütenmeer verwandeln. Später im Jahr ist jedoch von den meisten Arten nichts mehr zu erkennen. Was sind die Gründe dafür und vor allem wie überdauern diese Geophyten den Rest des Jahres?

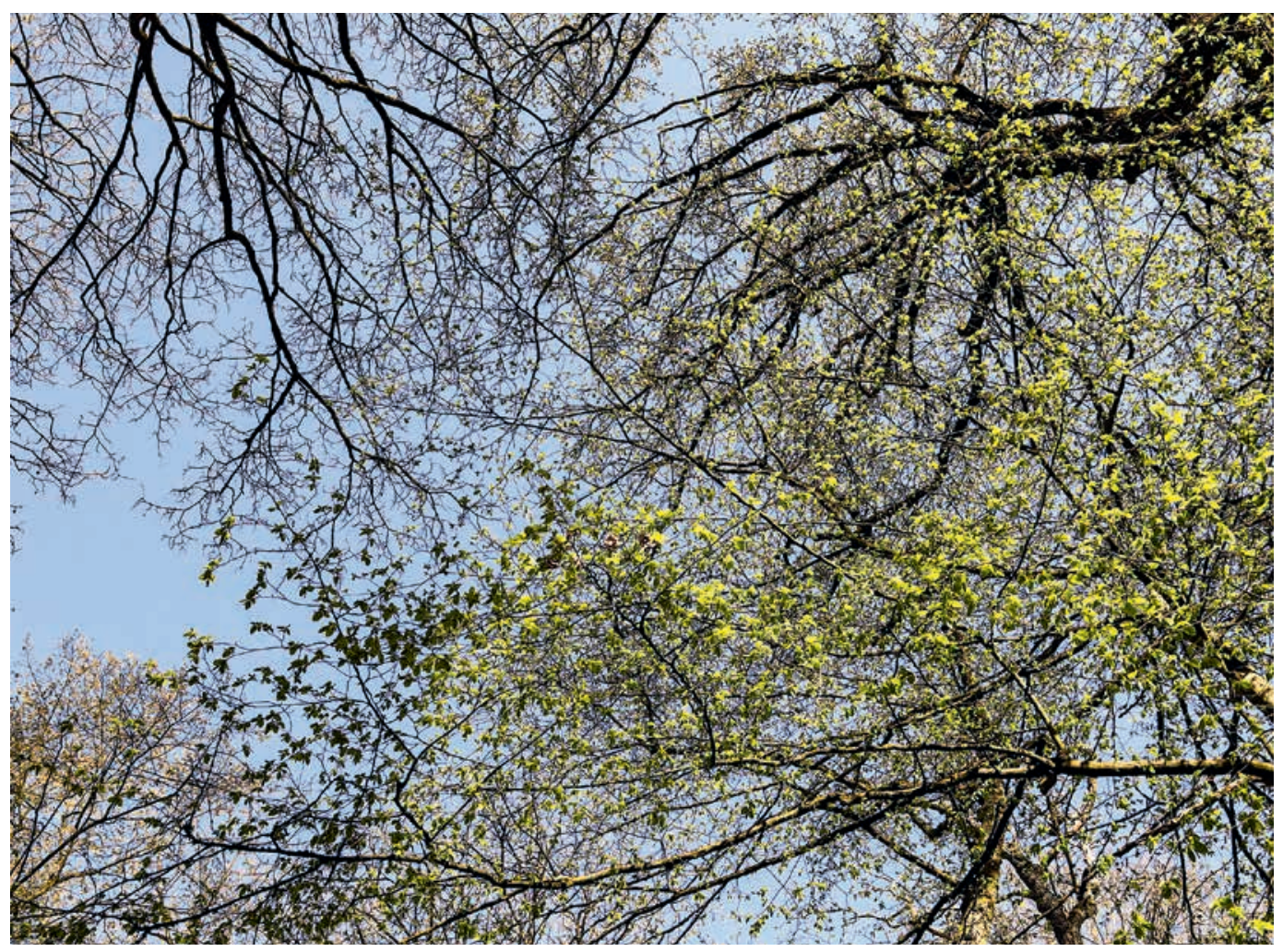

Abb. 1: Die kurze Zeit im Frühling, wenn es schon ausreichend warm ist und noch genug Licht durch die kaum belaubten Bäume auf den Waldboden fällt, ist die Zeit der Frühjahrsgeophyten. (Foto: H. Steinecke) 


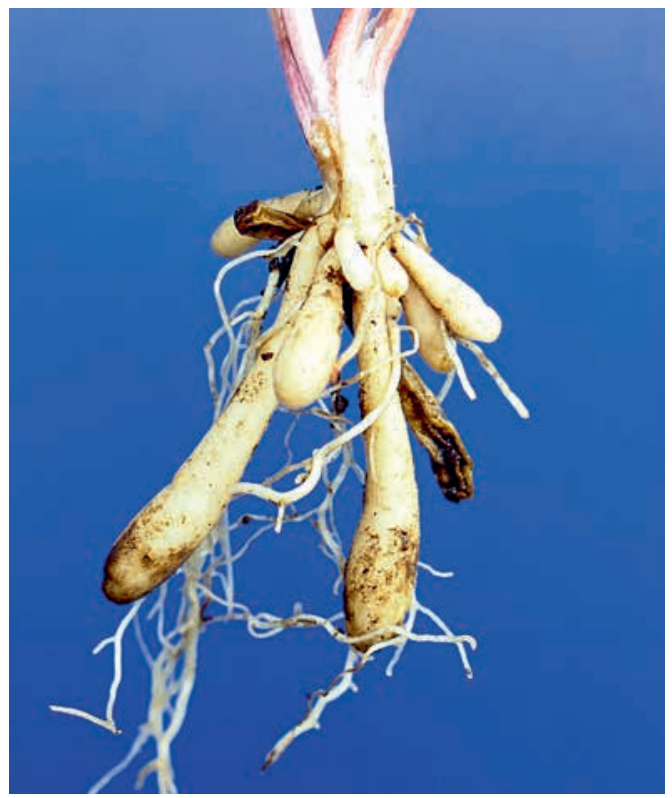

Abb. 2: Das Scharbockskraut überdauert den Winter mit Hilfe von Wurzelknollen. (Foto: V. M. DörkeN)

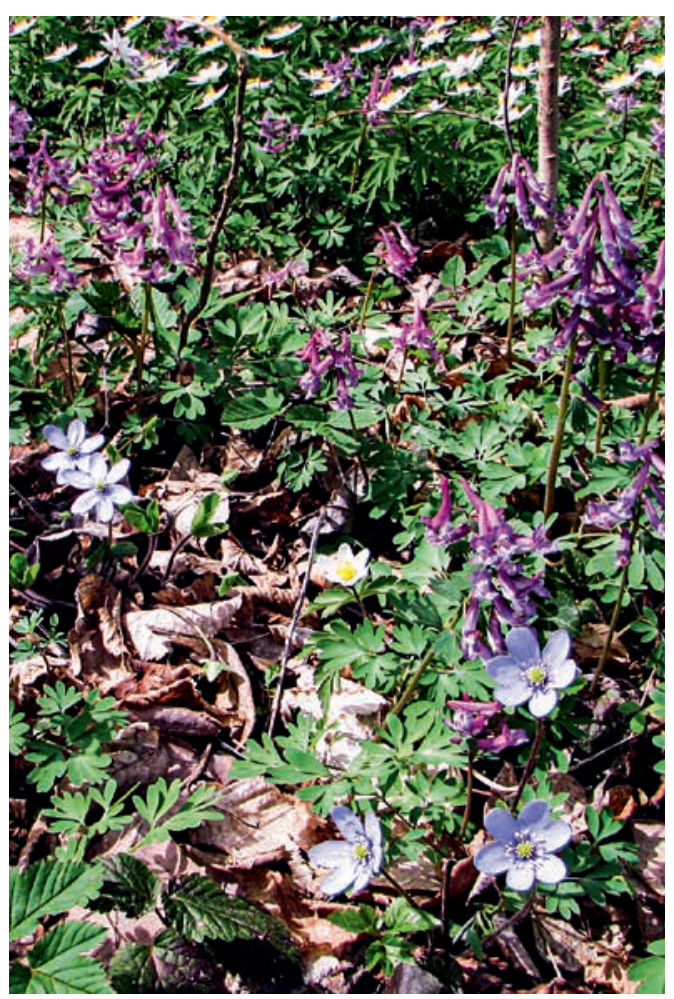

Abb. 3: Bunter Waldboden mit Leberblümchen, Buschwindröschen und Festem Lerchensporn auf dem Mägdeberg am Bodensee. (Foto: V. M. Dörken)
Die natürlichen Wälder Mitteleuropas (Ausnahme: Bergwälder der Alpen) werden größtenteils durch Laubbäume geprägt, allen voran die RotBuche (Fagus sylvatica). Im Herbst wird bekannter Weise das Laub abgeworfen, dies als Anpassung an winterliche Kälte mit Frost und Schnee. Im kommenden Frühjahr erfolgt dann artspezifisch, witterungsbedingt und abhängig von der Höhenlage der Neuaustrieb meist von Mitte April bis Mitte Mai. In belaubten und unbelaubten Wäldern herrschen ganz unterschiedliche Belichtungsverhältnisse, vor allem am Waldboden, vor. In der winterkahlen Phase ist der Wald lichtdurchflutet. Nach dem Laubaustrieb der Bäume trifft dagegen nur noch ein verschwindend geringer Bruchteil der eingestrahlten Sonnenenergie auf den Waldboden. Teilweise ist dies nur noch ein Prozent der ursprünglich einfallenden Strahlung. Das Blätterdach des Waldes fängt den Großteil der Strahlung $a b$, die die belaubten Bäume für eine effektive Photosynthese nutzen. Die Kronenschicht in den Wäldern ist ziemlich dicht, um die eingestrahlte Energie bestmöglich auszunutzen.

Wenn von Ende Oktober bis Mitte Mai der Waldboden hell ist und direkte Sonneneinstrahlung auf den Waldboden trifft, erwärmt sich dieser entsprechend. Das Frühjahr ist der Zeitraum, in dem höhere Temperaturen ein Wachstum in der Krautschicht erlauben, bis es nach voller Laubentwicklung der Bäume dort für die meisten Pflanzen zu dunkel ist.

\section{Geophyten nutzen die helle Zeit im Wald}

Dieser jahreszeitliche Wechsel der Lichtintensität am Waldboden hat in unseren heimischen Laubwäldern zu einem saisonalen Wechsel in der Vegetationsschicht geführt. So dominieren im zeitigen Frühjahr vielerorts Frühlingsgeophyten in der Krautschicht des Waldbodens. Frühlingsgeophyten sind Pflanzen, die mit einem unterirdischen Überdauerungsorgan den Winter überstehen. Durch den in diesen Organen eingelagerten Nährstoffvorrat können sie bereits zeitig austreiben, ohne auf große Photosyntheseleistung angewiesen zu sein. 
Überdauerungsorgane der Geophyten können Kriechsprosse (Rhizome), Zwiebeln oder Knollen sein. Die in ihnen eingelagerten Reservestoffe wurden in der vergangenen Vegetationsperiode während der belaubten Phase der entsprechenden Gewächse gebildet.

Der Großteil der Frühjahrsgeophyten hat einen ziemlich kurzen Lebenszyklus. Bereits kurz nach oder mit ihrem Austreiben erscheint auch schon die Blüte mit anschließender Fruchtbildung. Sie entwickeln schnell ihr Laub, betreiben Photosynthese und lagern Speicherstoffe ein. Viele haben bereits zum Zeitpunkt der Laubentfaltung der Bäume ihren jährlichen Lebenszyklus abgeschlossen. Die oberirdischen Teile der Pflanzen sterben nach der Fruchtreife ab, sie „ziehen ein.“ Unterirdisch gut geschützt überdauern sie bis zum zeitigen Frühjahr, bis der Waldboden wieder lichtdurchflutet ist und sich dann dort auch verschiedene Insekten, die als Bestäuber in Betracht kommen, aufhalten.

Vielerorts ist bereits ab Ende Mai nichts mehr von der zuvor herrschenden Blütenpracht und Artenvielfalt der Krautschicht des Waldes erkennbar. Im Sommer sind in den dunklen Wäldern oft nur noch wenige, extrem schattentolerante Arten wie Farne oder manche Süßgräser und Seggen zu finden.

\section{Heimische Frühlingsgeophyten}

Eine der bei uns am frühesten austreibenden und sehr häufigen Arten ist das Scharbockskraut (Ficaria verna), dessen Blätter teilweise schon im Februar erscheinen. Es ist besonders auf feuchten bis nassen Standorten in Au- und Bruchwäldern zu finden. Gerade im Frühjahr bilden die sternförmigen gelben Blüten ein wichtiges Nahrungsangebot für die ersten Insekten des Jahres. Die Perigonblätter sind kelchartig, während die Nektarblätter gelb und kronblattartig sind. Obwohl die Blüten häufig von Insekten aufgesucht werden, zeigt sich kaum Samenansatz. Das Scharbockskaut vermehrt sich vor allem vegetativ über Brutknollen, die in den Blattachseln meist nach der Blüte gebildet werden. Aufgrund dieser schafft es das Scharbockskraut, in kürzester Zeit teilweise riesige Populationen aufzubauen.

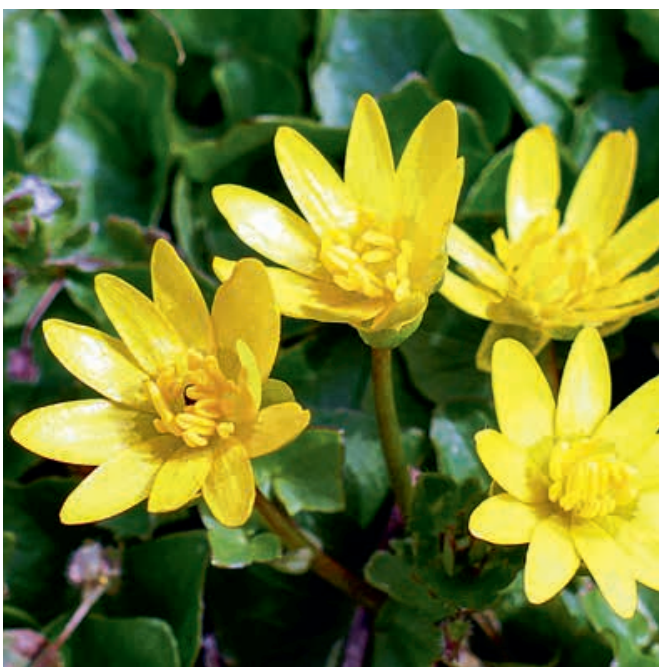

Abb. 4: Die gelben sternförmigen Blüten des Scharbockskrautes erscheinen oft in größeren Gruppen.

(Foto: H. Steinecke)

Wie der Name bereits andeutet, öffnet der Märzenbecher (Leucojum vernum), ein Zwiebelgewächs, schon ab Ende Februar bis in den März hinein seine glockenförmigen weißen Blüten. Wenn sie einmal geöffnet sind, schließen sie sich nicht mehr, so dass die weiße Blütenhülle bei

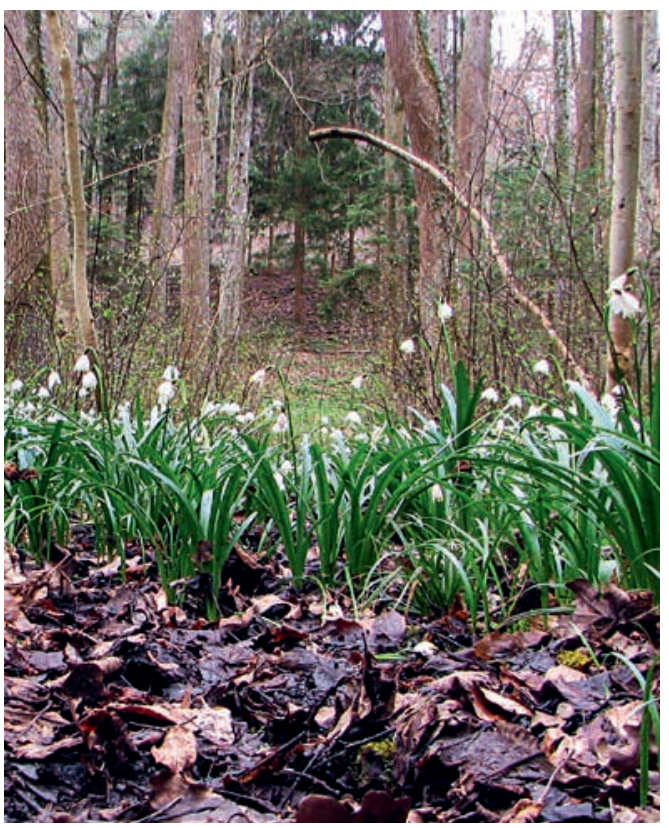

Abb. 5: Märzenbecher-Bestand in einem Wald im Bodenseegebiet. (Foto: V. M. DörkeN) 


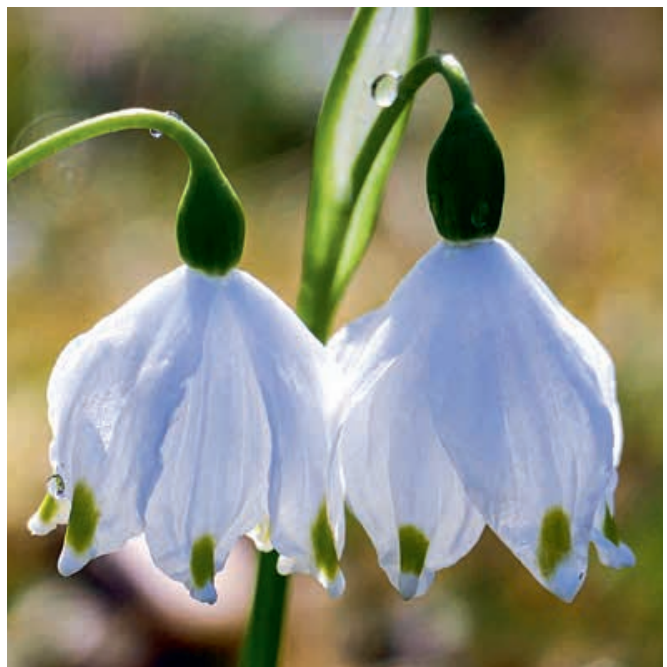

Abb. 6: Blüten des Märzenbechers. (Foto: H. STeinecke)

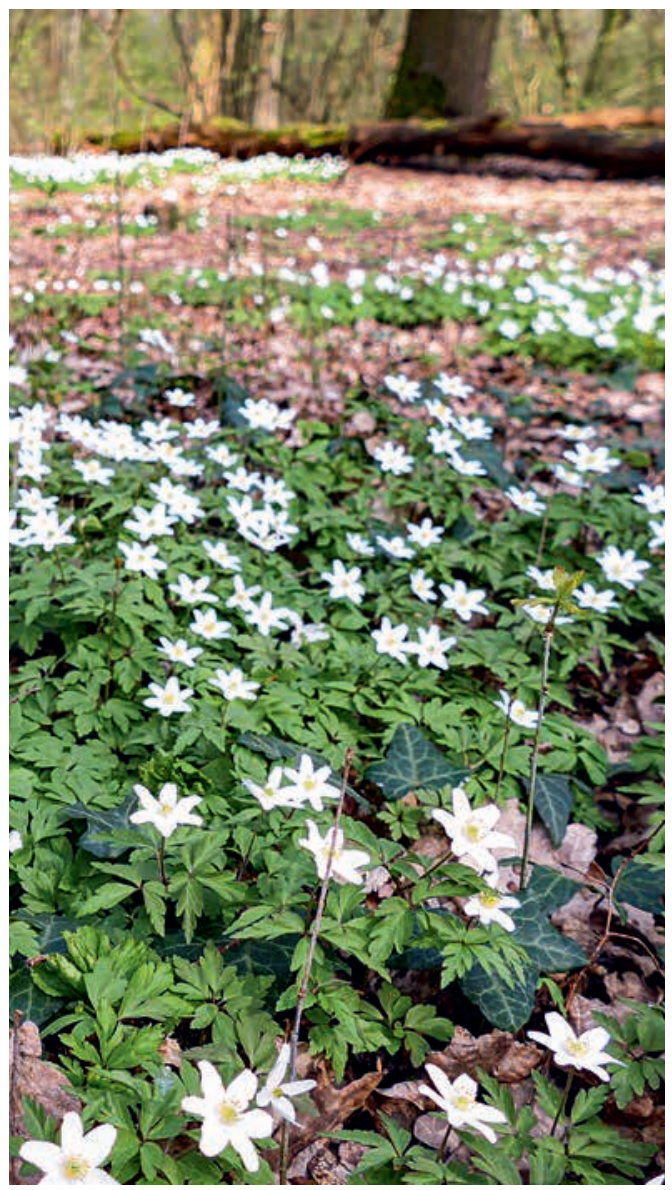

Abb. 7: Buschwindröschen im Ginnheimer Wäldchen in Frankfurt (Foto: H. STEINECKe)

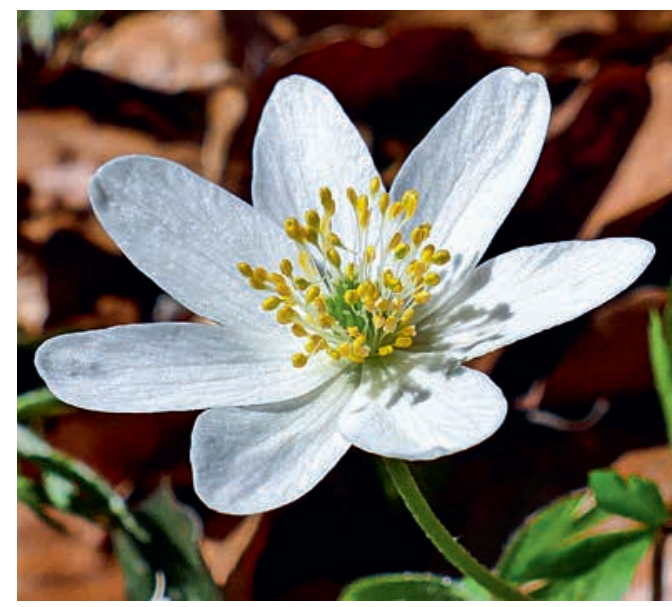

Abb. 8: Mit ihren vielen Staubblättern bietet die Blüte des Buschwindröschens vielen Insekten reichlich Pollennahrung an. (Foto: V. M. Dörken)

jedem Wetter eine recht große Fernwirkung hat. Märzenbecher sind in unseren Gärten wie auch die ähnlichen Schneeglöckchen beliebte Zierpflanzen. Beide Arten wachsen wild in Au-, Bruch- und feuchten Schluchtwäldern. Besonders im Bereich der hellgrünen Flecken an den Kronblattzipfeln entströmt den Blüten des Märzenbechers ein süßlicher Duft. Bienen und Tagfalter sind die wichtigsten Bestäuber. Am Samen befindet sich ein kleines fett- und proteinreiches Futterkörperchen (Elaiosom). Ameisen fressen diese energiereichen Elaiosomen, verschleppen dabei die Samen und tragen somit zur Ausbreitung bei.

Zu den wohl auffälligsten Frühlingsgeophyten in unseren heimischen Wäldern zählen das Buschwindröschen (Anemone nemorosa) und der Bärlauch (Allium ursinum). Während das Buschwindröschen als ein Rhizomgeophyt über ein ausgeprägtes, weitläufiges und reich verzweigten unterirdisches System aus Kriechsprossen verfügt, ist der Bärlauch eine Zwiebelpflanze. Die Zwiebel des Bärlauchs hat nicht die typische bauchige Form einer Zwiebel, sondern ist langestreckt und schmal. Im Rhein-Main-Gebiet gibt es stellenweise Massenvorkommen dieser Frühlingsboten. Manchmal wirkt der Waldboden zur Hauptblütezeit so, als hätte es geschneit, wenn sich die unzähligen weißen schalen- bzw. sternförmigen Blüten von Anemone nemorosa und Allium ursinum 


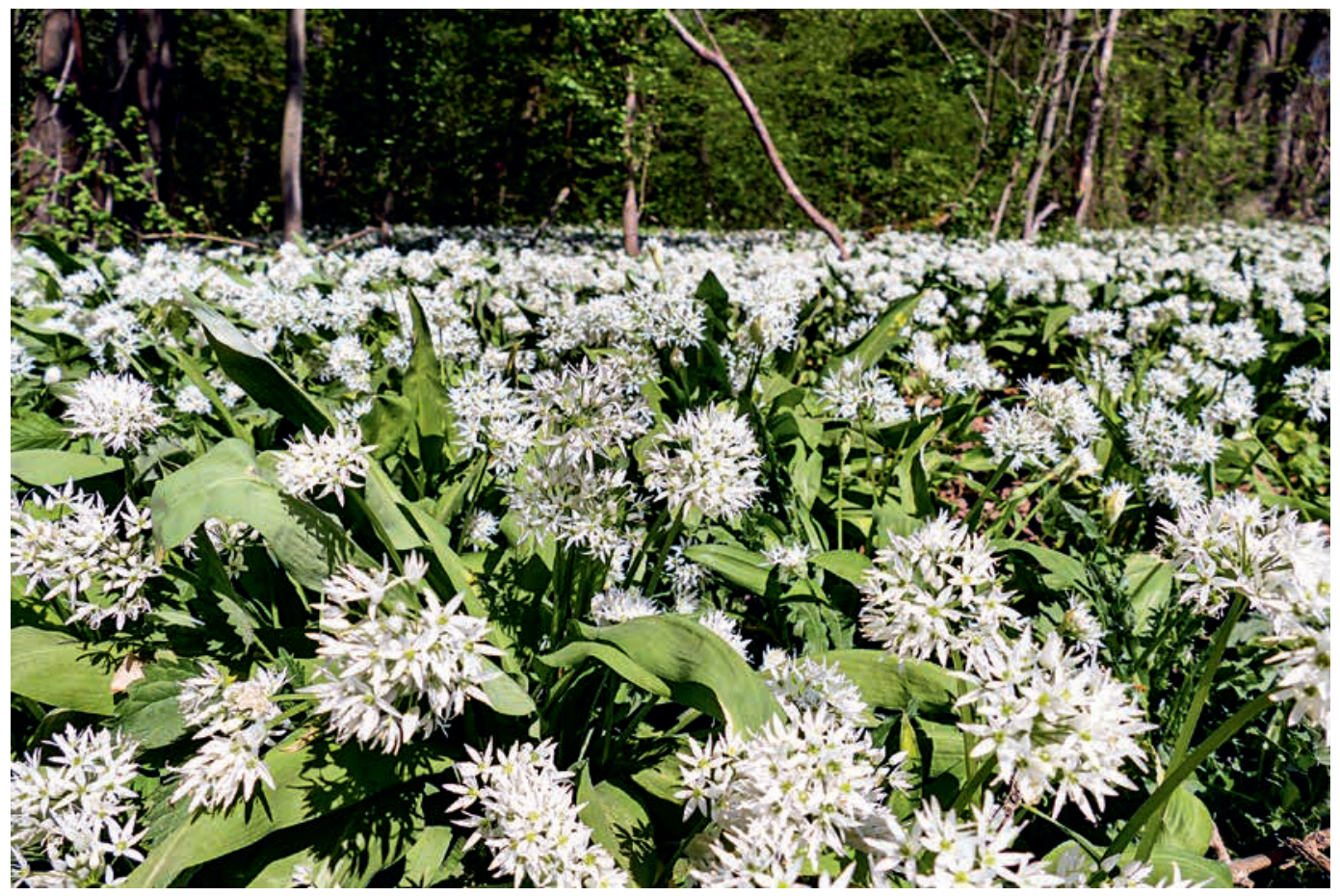

Abb. 9: Üppiger Bärlauch-Bestand im Bad Vilbeler Stadtwald. (Foto: H. Steinecke)

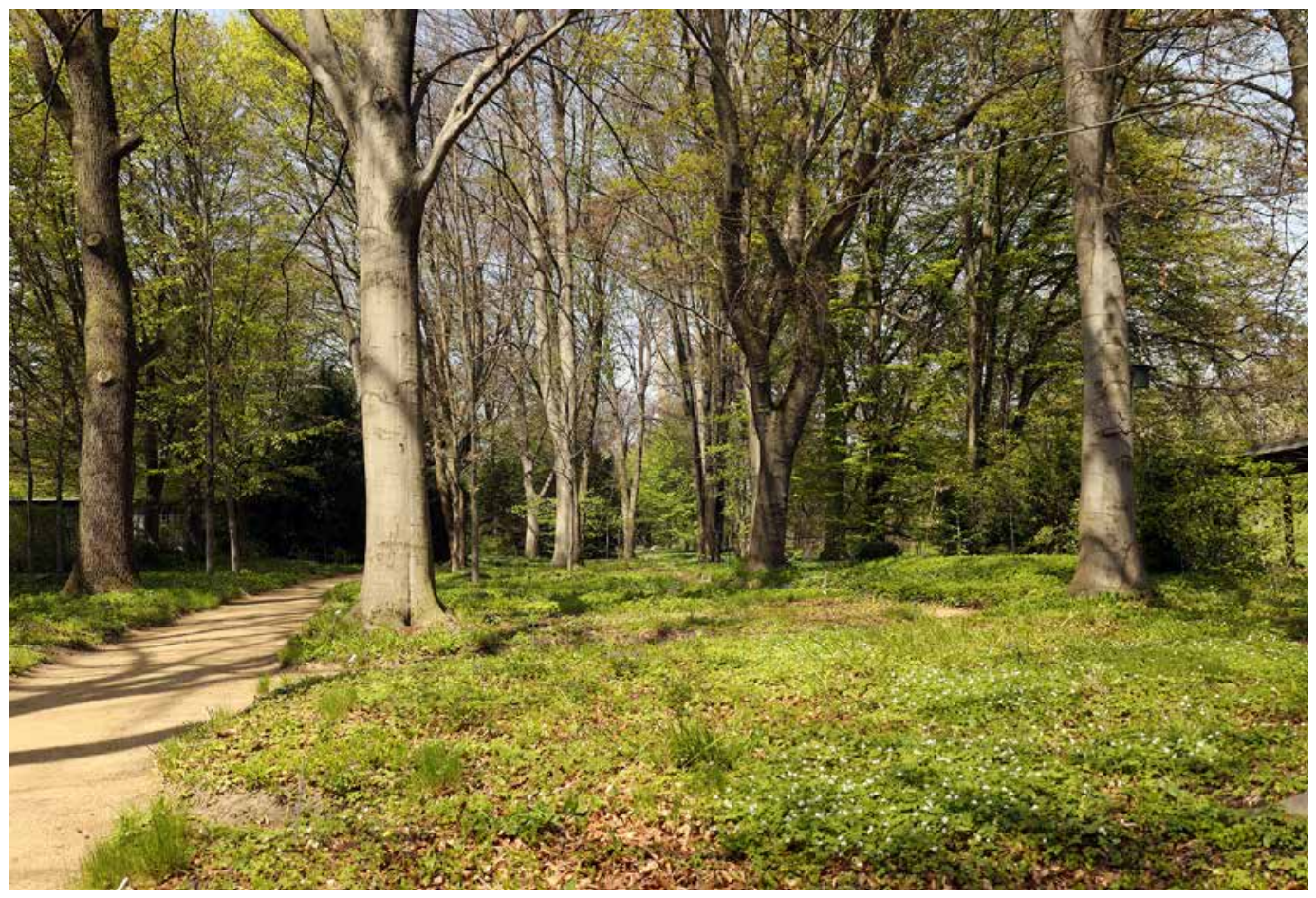

Abb. 10: Es wird Frühling im Buchenwald im Botanischen Garten Frankfurt. Nach dem Verblühen der Buschwindröschen erscheint in diesem Waldstück der Bärlauch. (Foto: H. Steinecke) 


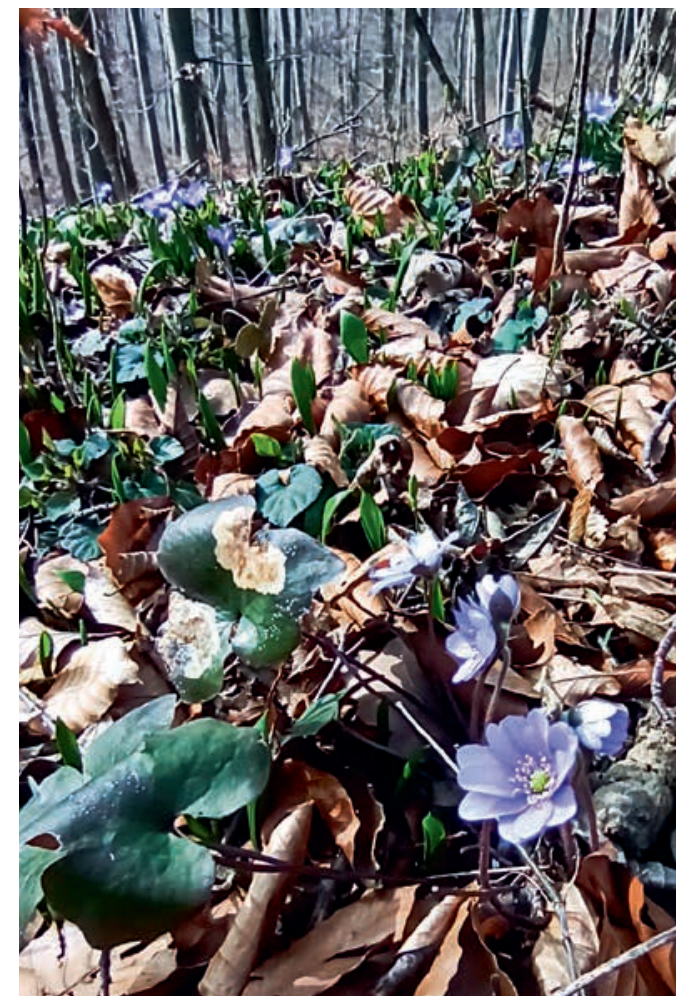

Abb. 11: Leberblümchen im Höhenzug Harly bei Goslar. (Foto: M. SснміDт)

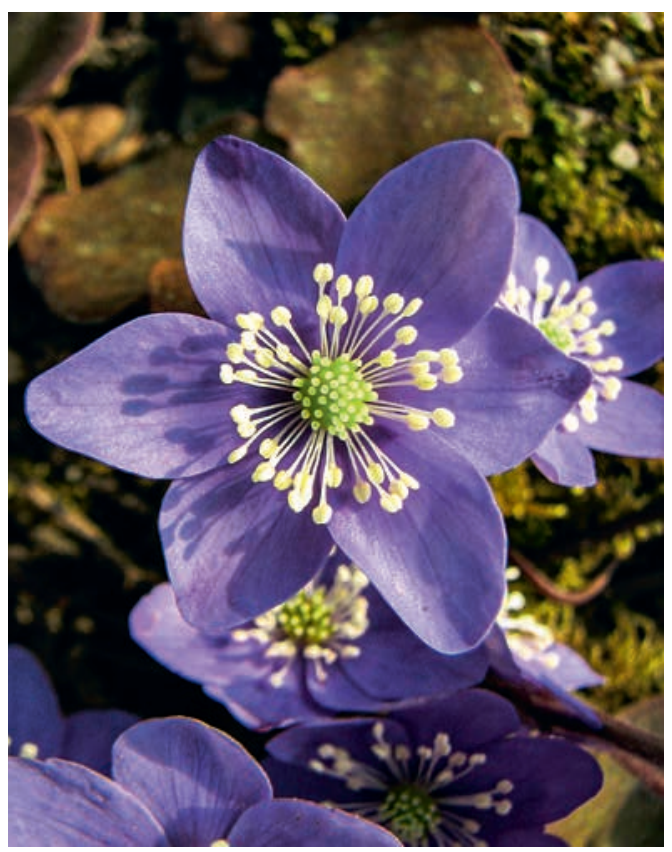

Abb. 12: Blüte eines Leberblümchens. (Foto: V. M. DöRkeN) geöffnet haben. Beispiele solcher geophytenreicher Wälder in und um Frankfurt sind der Niedwald, das Ginnheimer Wäldchen oder der Bad Vilbeler Stadtwald. Einen sehr schönen Buchenwald mit diversen Frühjahrsgeophyten gibt es auch im Botanischen Garten Frankfurt.

Die Pflanzen zeigen verschiedene Strategien, um einerseits gegenüber kalten Frühjahrstagen gewappnet zu sein, anderseits die doch eher noch in geringerer Individuenzahl fliegenden Insekten effektiv anzulocken. Das Buschwindröschen richtet seine Blüten so in Richtung Lichteinfall aus, dass sich die Blüten im Inneren aufwärmen. Mit diesem eng verwandt ist das Leberblümchen (Hepatica nobilis), das allerdings viel seltener ist und vor allem in Kalkbuchenwäldern vorkommt. Die schalenförmige Blüte wird schützend von drei kelchblattartigen Hochblättern umgeben. Zahlreiche Staubblätter stehen um die freien grünen Fruchtblätter, die an ihrer Spitze jeweils eine weiße Narbe tragen. Nektar wird nicht angeboten, die Bestäuber fressen den Pollen. Die Blüten schließen sich zur Nacht oder bei Regen und Kälte und während der Öffnungs- und Schließbewegungen wachsen die Blütenblätter, so dass die Blüten nach etwa einer Woche doppelt so groß geworden sind.

Etwa zeitgleich mit Buschwindröschen und Leberblümchen blüht auch der Lerchensporn (Corydalis), der ebenfalls großflächig individuenreiche Bestände mit weiß- und rosafarbig blühenden Individuen bilden kann. Während der Feste Lerchensporn (Corydalis solida) eine kompakte Knolle hat und die Tragblätter fingerförmig eingeschnitten sind, bildet der Hohle Lerchensporn (Corydalis cava) eine Knolle mit einem Hohlraum und ganzrandige Tragblätter. Lerchensporn-Blüten sind relativ lang gespornt. Insekten sind oft „faul“ oder haben einen zu kurzen Rüssel, um über den Blüteneingang an den Nektar zu gelangen. Sie beißen den Sporn an seiner Spitze auf und klauen den Nektar, ohne dabei zu bestäuben.

Eine typische Art in Buchenwäldern auf frischen, nährstoffreichen und kalkhaltigen Böden ist die Frühlings-Platterbe (Lathyrus vernus), die mit einem Rhizom den Winter überdauert. Ihre 


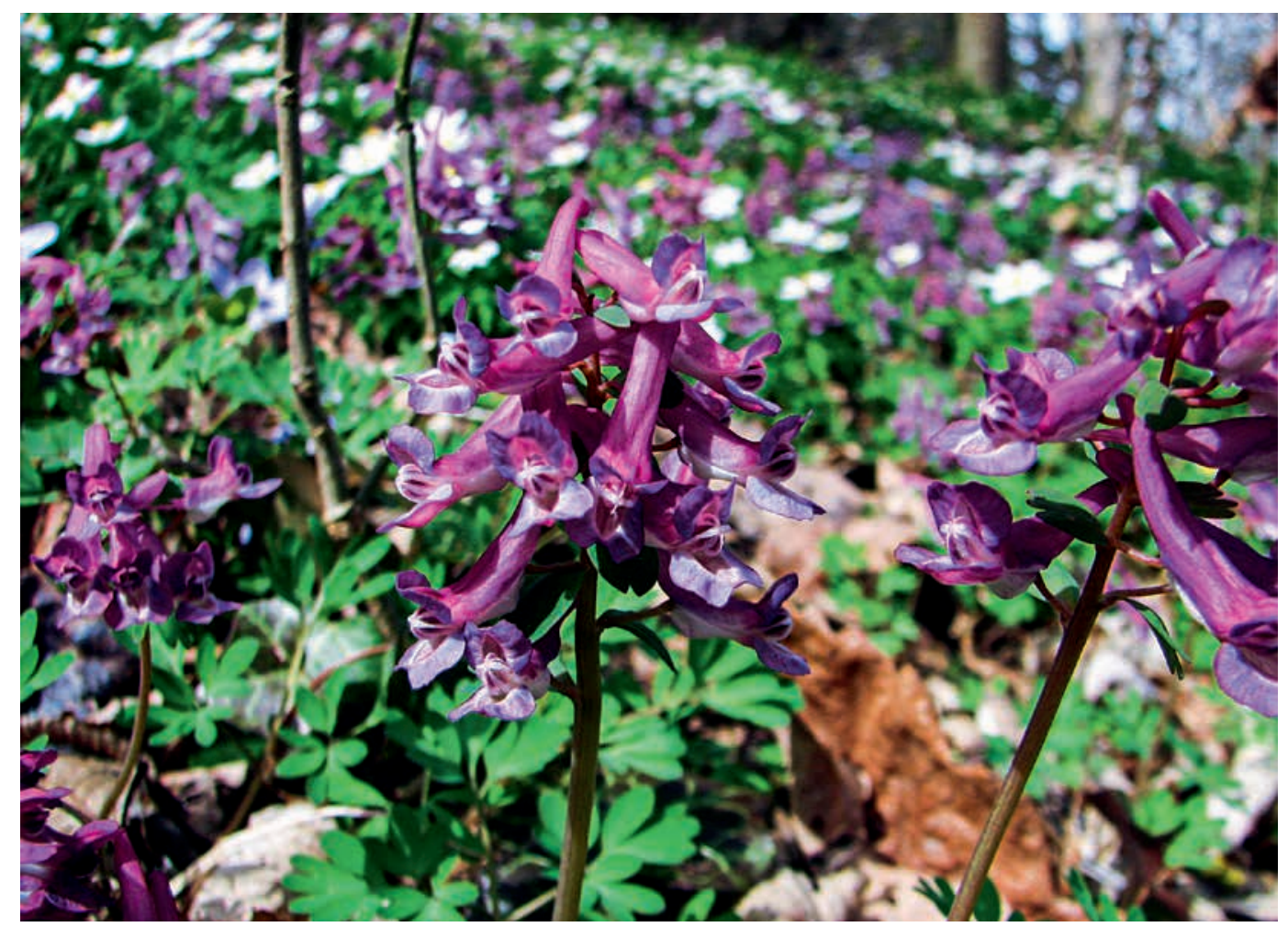

Abb. 13: Fester Lerchensporn in einem Wald am Bodensee. (Foto: V. M. DöRkeN)

Strategie, Bestäuber zielgerichtet anzulocken, besteht in einem Farbwechsel der Blüten. Junge Blüten sind rosa gefärbt, ältere nach der Bestäubung bläulich. Durch Veränderung des Säuregrades im Zellsaft verändert sich auch die Farbe der in den Blüten eingelagerten Anthocyane. Bestäuber, vor allem Hummeln, nehmen die pinkvioletten, nektarreichen Blüten besonders gut wahr und werden so mit Hilfe der Farbgebung auf die noch zu bestäubenden und gleichzeitig noch nektarreichen Blüten hingewiesen.

$\mathrm{Zu}$ den giftigsten heimischen Frühlingsgeophyten zählt der Aronstab (Arum maculatum). Hierbei handelt es sich um einen Knollengeophyten, der bei uns besonders in Kalkgebieten recht häufig anzutreffen ist. Er ist meist mit Buschwindröschen, Bärlauch, Maiglöckchen und auch Salomonssiegel vergesellschaftet. Im Unterschied zu den Blättern des Bärlauchs hat der Aronstab pfeilförmige Blätter. Zudem haben die Blätter des Aronstabs eine Netznervatur, was unter ein-

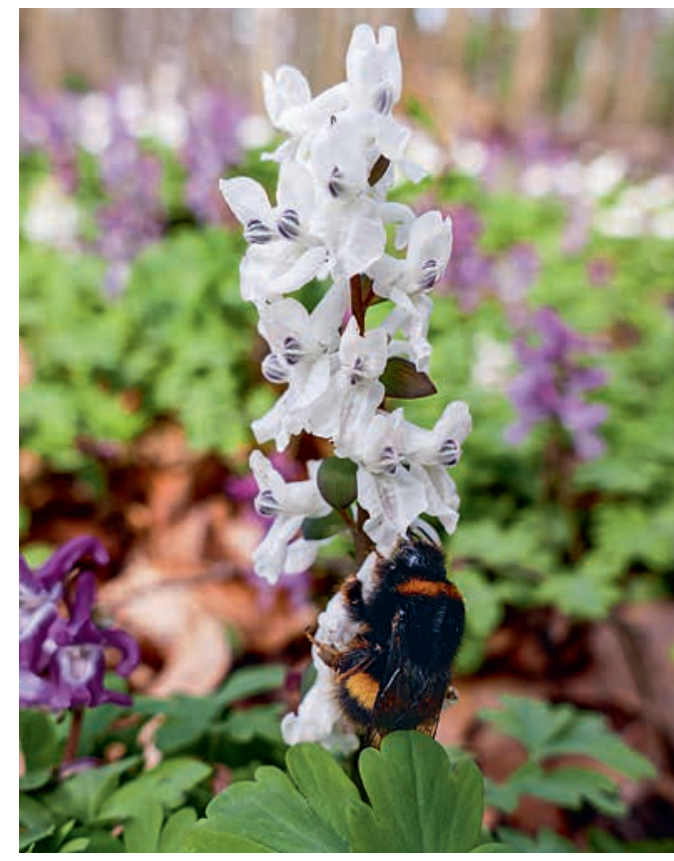

Abb. 14: Weißes Exemplar eines Festen Lerchensporns im Ginnheimer Wäldchen in Frankfurt. (Foto: H. Steinecke) 


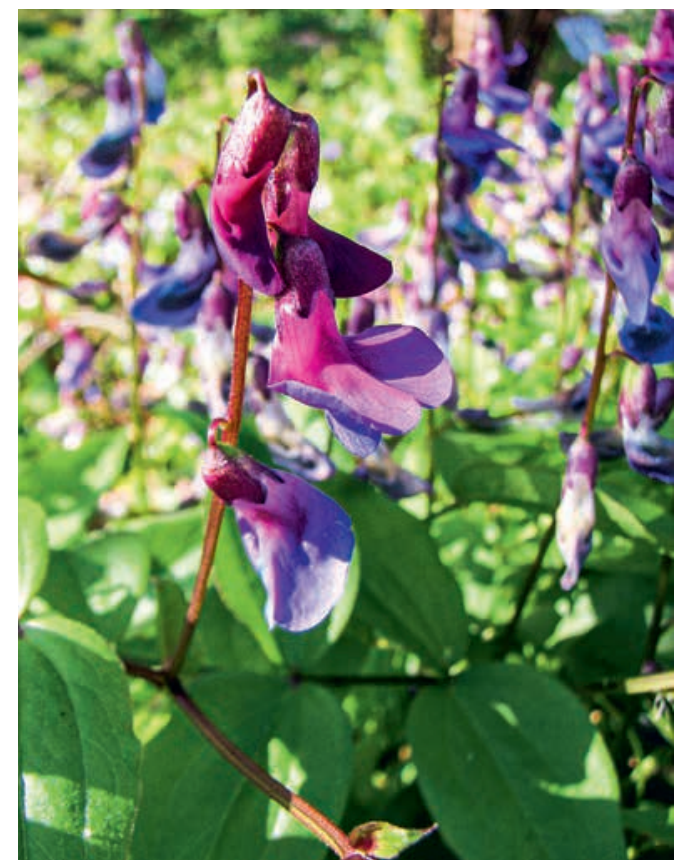

Abb. 15: Frühlings-Platterbse mit junger, rosa gefärbter Blüte und einer älteren, die bereits bläulich ist. (Foto. V. M. DörkeN)

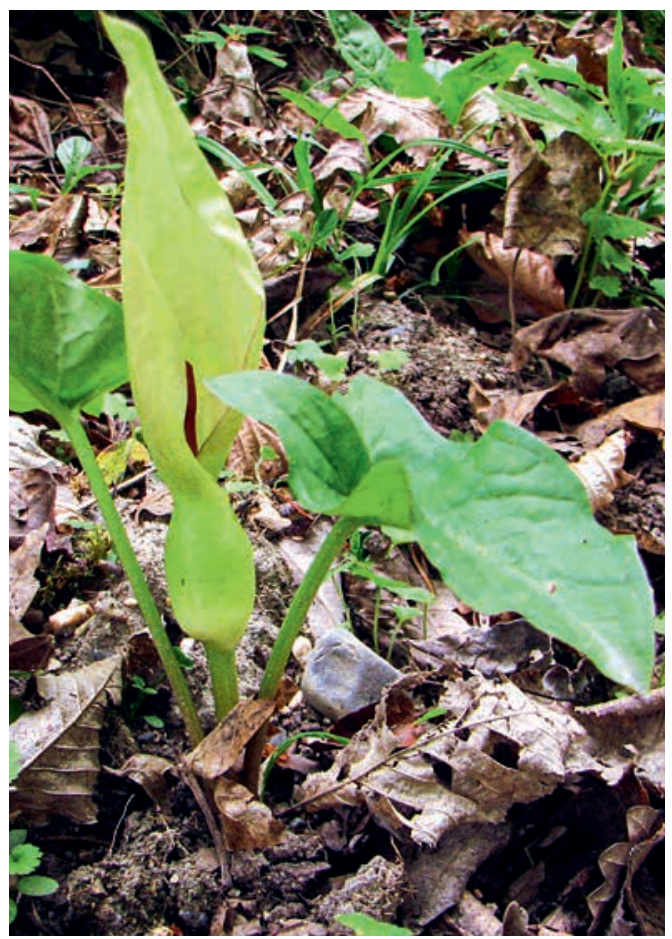

Abb. 16: Aronstab mit seinem charakteristischen Hochblatt, das den Kolben umgibt. (Foto. V. M. Dörken) keimblättrigen Pflanzen (dazu auch die Gräser) ein seltenes Merkmal ist. Bärlauch dagegen weist die für einkeimblättrige Pflanzen typische Parallelnervatur auf. So kann man beide Arten auch im nicht blühenden Stadium leicht voneinander unterscheiden, was aufgrund der Giftigkeit des Aronstabs besonders beim Bärlauch-Sammeln in Mischbeständen wichtig ist. Der Blütenstand des Aronstabs ist eine Kesselfalle. Hier stehen die Einzelblüten an der Basis einer aufrechten Blütenstandsachse und sind von einem weißen, auffälligen Hochblatt (Spatha) umgeben. Aus den Blüten des Aronstabs entwickeln sich dann später orange-rote Beerenfrüchte

Bei uns ein eher seltener Frühlingsgeophyt ist die Einbeere (Paris quadrifolia), die vor allem in basenreichen Wäldern anzutreffen ist. Sie ist ebenfalls eine einkeimblättrige Pflanze. Hier werden an den aufrechten Sprossachsen meist nur 4, seltener auch 5 oder sogar 6 Blätter wirtelartig gebildet. Eine Besonderheit dieser Blätter ist, dass diese wie die des Aronstabs eine Netznervatur aufweisen. Am Ende der Sprossachse zeigt sich dann eine einzelne Blüte. Diese ist unscheinbar mit gelben unauffälligen Blütenhüllblättern. Die Schauwirkung geht von den Staubblättern und dem schwarzvioletten Fruchtknoten aus. Aus der Blüte entwickelt sich nach erfolgreicher Bestäubung und Befruchtung eine einzige bläulich-schwarze auffällige Beere, worauf sich der Artname bezieht.

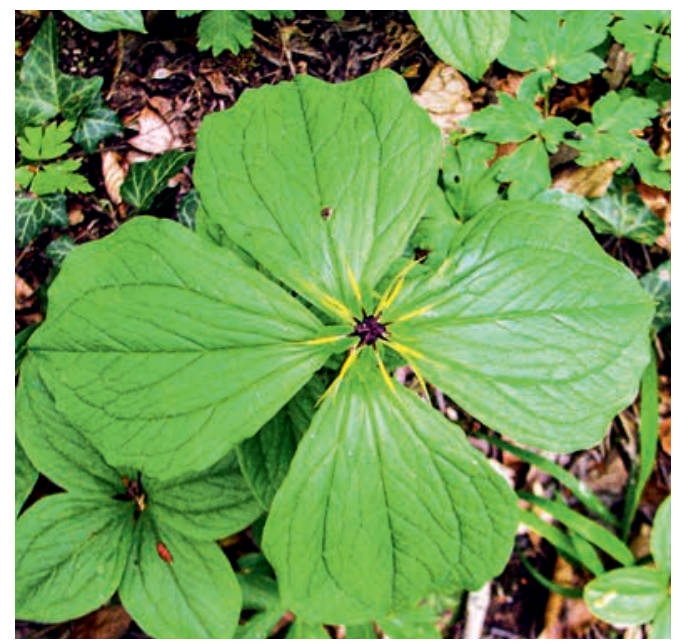

Abb. 17: Blühende Einbeere. (Foto: V. M. Dörken) 


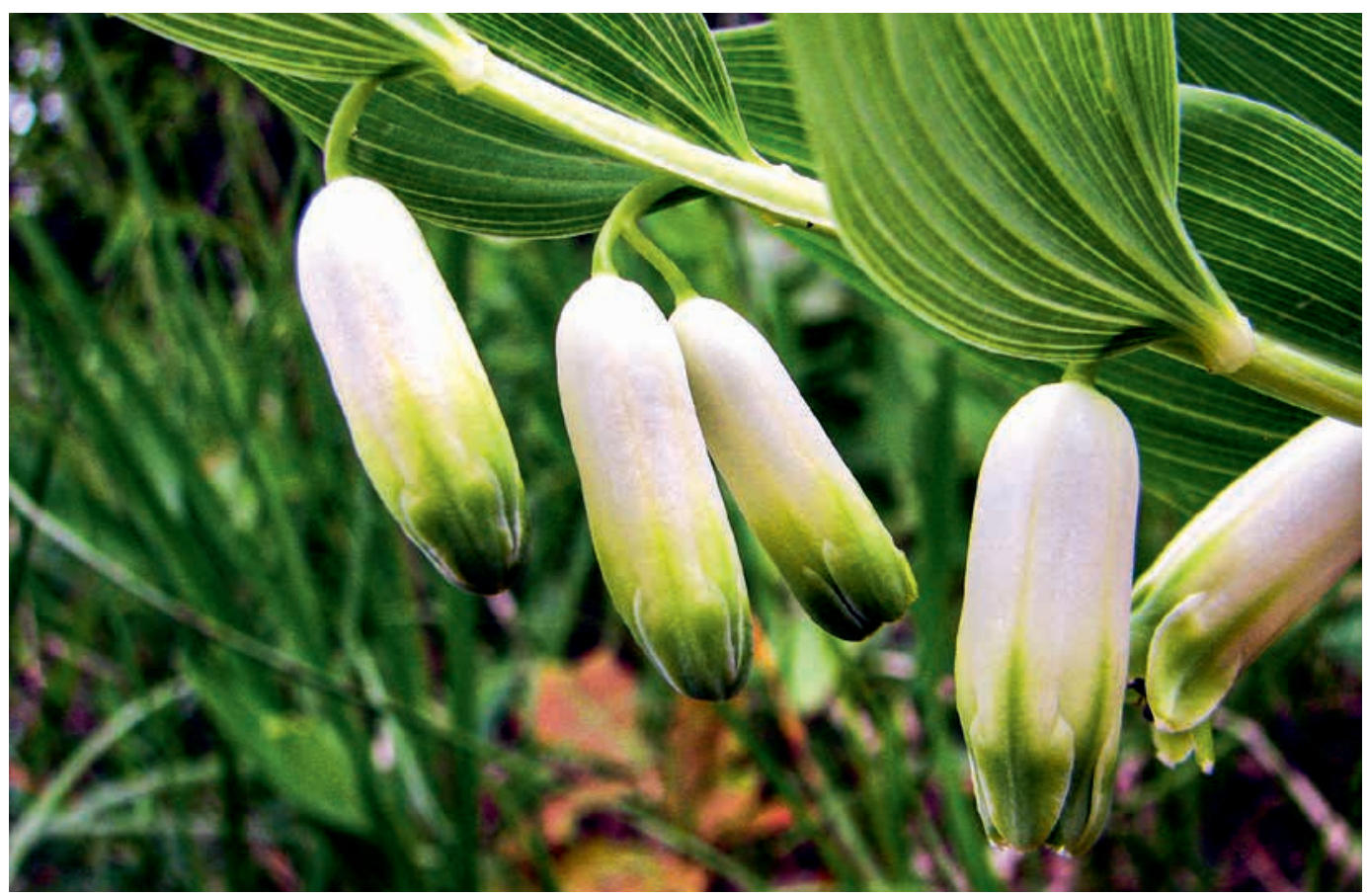

Abb. 18: Blüten des Wohlriechenden Salomonssiegels. (Foto: V. M. DörkeN)

Oft Zusammen mit der Einbeere wächst auch das Salomonssiegel (Polygonatum). Hierbei handelt es sich um einen Rhizomgeophyten. An seiner langen, schräg aufsteigenden Sprossachse sind die wechselständigen Blätter fischgrätenartig angeordnet. Auf der Unterseite werden die weißen Blüten in hängenden Blütenständen

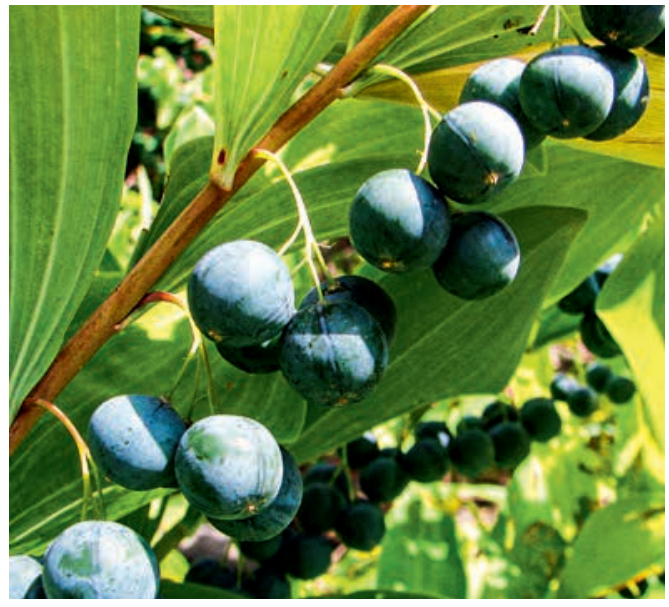

Abb. 19 : Früchte des Vielblütigen Salomonssiegels.

(Foto: V. M. Dörken) hervorgebracht, beim Vielblütigen Salomonssiegel (Polygonatum multiflorum) stehen in der Regel mehr als zwei Blüten zusammen, beim Wohlriechenden Salomonssiegel (Polygonatum odoratum) hingegen nur zwei, seltener auch nur eine. Die Blüten von Polygonatum odoratum duften, diejenigen von P. multflorum nicht. Nektar ist in den glockenförmigen Blüten verborgen. Er wird von langrüsseligen Hummeln und selten von Schmetterlingen aufgenommen. Neben Insektenbestäubung ist hier Selbstbestäubung keine Seltenheit. Aus den Blüten entwickeln sich dann später bläulich-schwarz bereifte Beeren.

\section{Anschrift des Autors und der Autorin}

PD Dr. Veit Martin Dörken, Universität Konstanz, Fachbereich Biologie, Universitätsstraße 10, 78457 Konstanz, E-Mail: veit.doerken@uni-konstanz.de; https://orcid.org/0000-0002-8451-6893

Dr. Hilke Steinecke, Palmengarten Frankfurt, Siesmayerstr. 61, 60323 Frankfurt, E-Mail: hilke.steinecke@stadt-frankfurt.de; https://orcid.org/0000-0002-4618-4867 